Military Technical College Kobry El-Kobbah, Cairo, Egypt

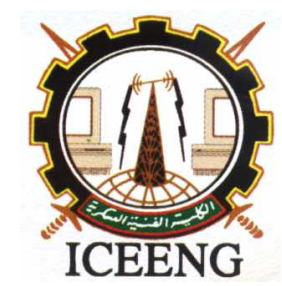

\author{
$6^{\text {th }}$ International Conference \\ on Electrical Engineering \\ ICEENG 2008
}

\title{
Surface and leaky wave propagation on planar finite dipole antenna arrays
}

By
D.F. El-Hossary *
E. A. El-Diwany **
H. H. El-Tamaly $* * *$

\section{Abstract:}

The active impedances and the currents of the elements in planar finite phased arrays of dipoles show the presence of scan dependent interference pattern between more than one wave. The first wave is the forced scanned space wave excited by the generators (or the wave corresponding to an incident plane wave) and the other waves are traveling waves on the planar dipole antenna array surface. The phenomenon of traveling waves on the array surface is investigated by searching for natural waves that can propagate on an infinite dipole array, where the natural waves are obtained as the source-free solution of the infinite array problem. The study reveals the presence of surface and leaky waves of different types. The effects of the array parameters on these traveling waves are investigated. These traveling waves are found to interpret many of the interference phenomena encountered.

\section{Keywords:}

Phased antenna arrays, planar dipole arrays, interference patterns on dipole arrays, surface waves, leaky waves.

\footnotetext{
* Brig. Gen.(R) Dr., Egyptian Armed Forces

** Assoc.Prof., Dr., Electronics research institute.

*** Prof. Dr., Faculty of Engineering, Minia University.
} 


\section{Introduction:}

The active impedances and the currents of the elements in planar finite phased arrays of dipoles show the presence of scan dependent interference pattern between more than one wave, in both the radiation problem and the problem of an incident plane wave. The first wave is the forced scanned space wave excited by the generators (or the wave corresponding to the incident plane wave) and the other waves are traveling waves on the planar dipole antenna array surface $[1,2]$. For small inter-element spacings and low source impedance, the traveling wave was identified as a surface wave similar to that propagating on linear Yagi-Uda arrays [2, 3]. Similar interference patterns are also found with half wavelength inter-element spacing and significant source impedance [1], which can not be interpreted as surface waves. The surface wave does not appear in the excitation of the infinite array, but becomes excited as a natural wave of the structure excited by the discontinuities at the ends of a finite array [2]. As other slow surface waves, such surface waves do not radiate as they propagate inside the array, but as the surface wave reaches the finite array end, it radiates in the endfire direction and partially reflects, causing similar phenomenon as it encounters the other edge, in a series of multiple reflections. Such surface waves cause scattering and radiation in unwanted directions. The phenomenon of traveling waves on the array surface can be investigated by searching for natural waves that can propagate on an infinite dipole array, where the natural waves are obtained as the source-free solution of the infinite array problem [2, 4]. This condition is equivalent to setting the dipole self impedance or admittance to zero. This condition allows natural currents (voltages) to exist on the antenna without exciting voltage (current). The present study reveals the presence of surface and leaky waves of different types. The effects of the array parameters on these traveling waves are investigated.

\section{Analysis of the array dipole currents and impedances}

Let us consider a finite array of M X N dipole elements with the dipoles oriented in the $\mathrm{x}$ - direction. The feed network of each dipole consists of a voltage source in series with a source impedance $Z_{s}$. The equations for the applied voltages for the dipoles in the array can be written in terms of the array currents, self, mutual impedances $Z_{i j}$ and source impedances $Z_{s}$ as $[1,2,5,6]$ :

$$
\begin{aligned}
& V_{1}=\left(Z_{11}+Z_{S}\right) I_{1}+Z_{12} I_{2}+--------+Z_{1 n} I_{n} \\
& V_{2}=Z_{21} I_{1}+\left(Z_{22}+Z_{s}\right) I_{2}+--------+Z_{2 n} I_{n} \\
& ---------------------------------\bar{n} \\
& V_{n}=Z_{n 1} I_{1}+Z_{n 2} I_{2}+--------+\left(Z_{n n}\right) I_{n}
\end{aligned}
$$


The array element currents are the solution of this matrix equation. The phase of the driving voltage in the $\mathrm{m}^{\text {th }} \mathrm{n}^{\text {th }}$ element in the array is expressed in terms of the scan angles $\Theta_{0}$ and $\varphi_{0}$ as:

$V_{m n}=A_{m n} \exp \left(-j \alpha_{0} k x_{m}-j \beta_{0} k y_{n}\right)$

where

$\alpha_{0}=\sin \theta_{0} \cos \phi_{0}, \quad \beta_{0}=\sin \theta_{0} \sin \phi_{0}, \quad k=2 \pi / \lambda, \quad \lambda$ is the wavelength

$\left(x_{m}, y_{n}\right)$ position of the $(\mathrm{m}, \mathrm{n})$ element, $x_{m}=m d_{x}$, and $y_{n}=n d_{y}$

The current on either dipole is assumed to have a sinusoidal distribution, namely for dipole 2;

$I_{2}\left(\zeta_{2}\right)=I_{m 2} \sin k\left(l_{2}-\left|\zeta_{2}\right|\right)$

The mutual impedance between two dipoles with sinusoidal current distribution is given as $[7,5,6]$

$\mathrm{Z}_{21}=\frac{1}{\sin k l_{1} \sin k l_{2}} \int_{-l 2}^{l 2} \frac{E_{x}\left(\zeta_{2}\right)}{I_{m_{1}}} \sin k\left(l_{2}-\left|\zeta_{2}\right|\right) d \zeta_{2}$

The vertical field component $\mathrm{E}_{\mathrm{x}}$ due to dipole 1 at dipole 2 can be written as [7]

$$
E_{x}=-j 30 I_{m}\left(\frac{e^{-j k k_{1}}}{r_{1}}+\frac{e^{-j k r_{2}}}{r_{2}}-2 \cos k l_{1} \frac{e^{-j k r}}{r}\right)
$$

where the distances $\mathrm{r}, \mathrm{r} 1, \mathrm{r} 2$ are from the observation point to the center and the ends of dipole 1, respectively. Substituting Eqn. (5) in Eqn. (4),

$Z_{21}=\frac{j 30}{\sin k l_{1} \sin k l_{2}} \int_{-l 2}^{l 2}\left(\frac{e^{-j k r_{1}}}{r_{1}}+\frac{e^{-j k r_{2}}}{r_{2}}-2 \cos k l_{1} \frac{e^{-j k r}}{r}\right) \cdot \sin k\left(l_{2}-\left|\zeta_{2}\right|\right) d \zeta_{2}$

This formulation, Eqn.(1), can be used both for an array excited by voltage sources to scan the array beam [1] (as shown above), or for scattering from an array with load impedances [2], with the array excited with an incident plane wave from the same direction as the scan angle. The impedance matrix may generally be obtained using a moment method solution of electric field integral equations on the elements [2]. For dipoles near half wavelength long, the current distribution on the elements is known to be fairly described by a sinusoidal function. The use of this distribution as a single entire domain expansion and weight function in the moment solution reduces the impedance matrix to the matrix of the self and mutual impedances of the elements, Eqns. $(1,6)$. In these cases the elements of the voltage vector, Eqn. (2), become the electric field in the exciting gap of the dipole (or the incident plane wave electric field) multiplied by the weight function (sinusoidal) and integrated on the dipole length. The case of the incident plane wave leads to Eqn. (1), for the fed dipoles, multiplied by a constant, with the scan angle equal to the angle of incidence of the plane wave. 


\section{Interference patterns on dipole arrays}

Numerical calculation of the active impedances and excited currents is made for cylindrical dipoles. Calculated currents are studied for the case of an incident plane wave in H-plane at incidence angle $\theta_{0}=45^{\circ}$. Cylindrical dipole length and radius are $0.39 \lambda$ and $0.006 \lambda$, respectively, element spacings are $0.41 \lambda$ and $0.23 \lambda$ in $x-$ and $y-$ directions, respectively. Figure 1 shows the element currents for $25 \mathrm{x}$ infinite array for incident plane wave at angle $\theta_{o}=45^{\circ}$ with zero load impedance [2]. Fig. 2 shows the corresponding computed results for $25 \times 25$ finite array, which agree reasonably (a scaling factor exists due to different amplitudes of the incident wave). Fig. 3 shows the spectrum of the computed current of Fig. 2, (the unique range of the spectrum for such periodic current of period $\mathrm{d}_{\mathrm{y}}$ is from $-\lambda / 2 \mathrm{~d}_{\mathrm{y}}$ to $\lambda / 2 \mathrm{~d}_{\mathrm{y}}$, which in the present case becomes in the range $-2.17<\beta<2.17$ ). The spectrum shows the existence of three propagating waves, one is the forced wave excited by the incident plane wave with $\beta=\operatorname{Sin} \theta$, the other is a surface wave with $\beta \cong 1.25$, and the third is the corresponding surface wave propagating in the reverse direction (reflected at the array end), where $\beta$ is normalized w.r.t. 'k'. Figure 2 shows an interference pattern along the array between these waves. If we have (without loss of generality) two propagating waves of equal amplitudes; $e^{j \beta_{1} y} \& e^{j \beta_{2} y}$, their sum becomes,

$E=e^{j \beta_{1} y}+e^{j \beta_{2} y}$

$$
=2 \cos \left(\frac{\beta_{1}-\beta_{2}}{2}\right) y e^{j\left(\frac{\beta_{1}+\beta_{2}}{2}\right) y}
$$

The exponential term $e^{j\left(\frac{\beta_{1}+\beta_{2}}{2}\right) y}$ represents a traveling wave with propagation constant $\left(\frac{\beta_{1}+\beta_{2}}{2}\right)$, and the cosine term represents a standing wave pattern along the array. It is to be noted that a half cycle in the cosine interference pattern corresponds to a complete cycle seen in the standing wave pattern on the array since both negative and positive half cycles in the cosine pattern become an envelope for the traveling wave, thus the period ' $\mathrm{y}_{\mathrm{c}}$ ' of complete cycle in the standing wave pattern on the array is obtained from the following condition, where $\beta_{1}$ and $\beta_{2}$ are normalized w.r.t. $\mathrm{k}$ :

$$
\begin{aligned}
& \frac{2 \pi}{2 \lambda}\left(\beta_{1}-\beta_{2}\right) y_{c}=\pi \\
& \text { or } \frac{y_{c}}{\lambda}=\frac{1}{\beta_{1}-\beta_{2}}
\end{aligned}
$$

Active impedance results are computed with (51 x 51) elements array, Fig. 4, for scan in H-plane at $60^{\circ}$. Cylindrical dipole length and radius are $0.486 \lambda$ and $0.002 \lambda$, respectively, which correspond to the resonant length in an infinite array. The impedances again show an interference pattern. 

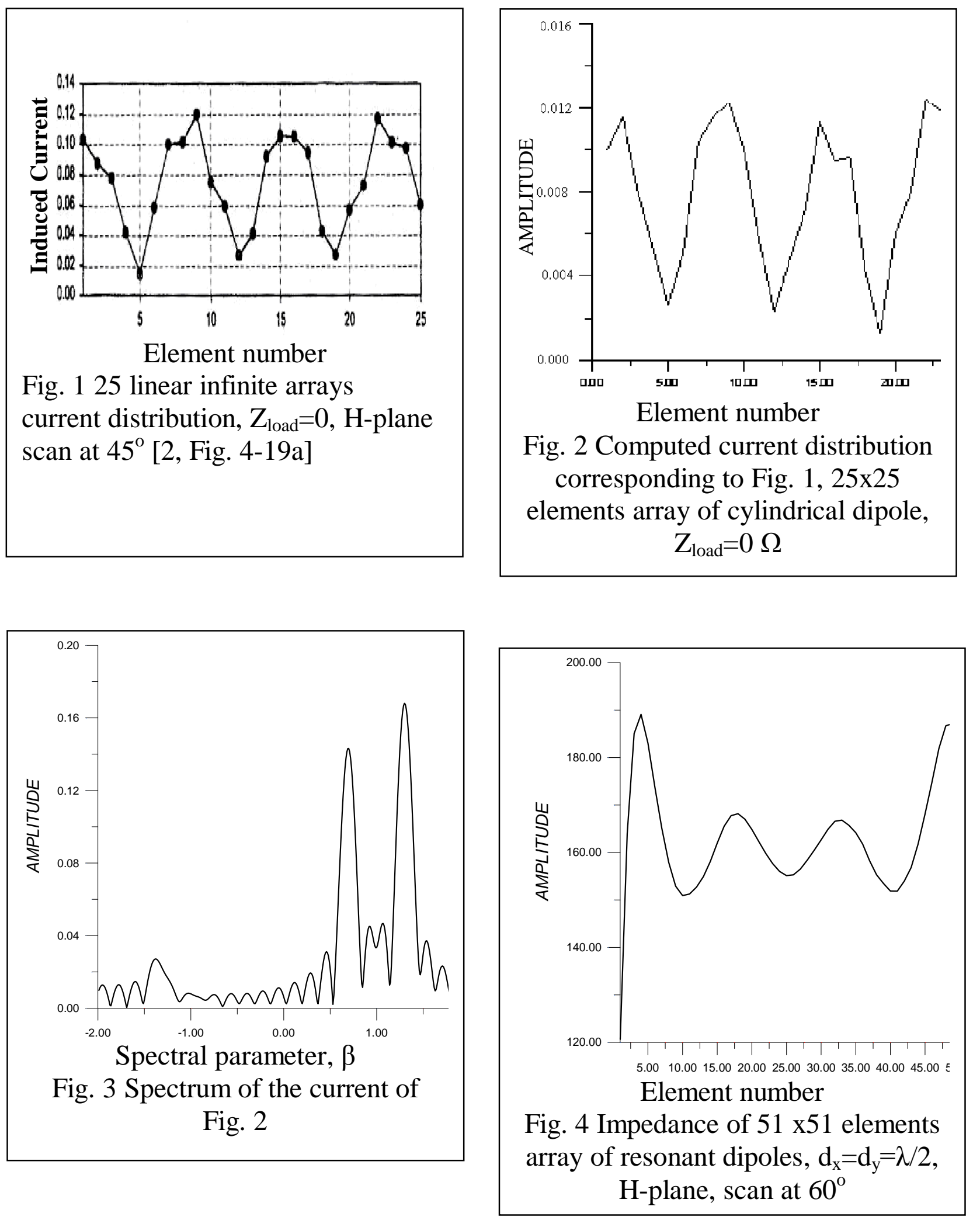


\section{Traveling waves on infinite planar dipole arrays and interpretation of interference patterns.}

The propagation constant of the surface wave $(\beta)$ propagating on the planar finite by infinite array was obtained by applying the condition that the active impedance of the dipole in an infinite array is equal to zero, which corresponds to the source-free solutions for wave propagation on the array, since current can be supported with zero voltage $[2,8]$. In what follows we investigate the different types of natural traveling waves on the surface of an infinite array of strip dipoles.

\section{Natural traveling waves on an infinite planar array of strip dipoles}

The active impedance of a strip dipole in an infinite planar array of strip dipoles of length ' $a$ ' and width ' $b$ ' and separations $d_{x}, d_{y}$ in the $x$ - and $y$-directions, respectively, is given by $[9,5]$,

$$
Z_{P}(\alpha, \beta)=\frac{1}{d_{x} d_{y} k^{2}} \sum_{m} \sum_{n} \bar{Z}\left(\alpha+\frac{\lambda}{d_{x}} m^{\prime}, \beta+\frac{\lambda}{d_{y}} n^{\prime}\right)
$$

where $\bar{Z}$ is given by:

$$
\begin{aligned}
& \bar{Z}\left(\alpha_{f}, \beta_{f}\right)=960 \pi\left(\frac{a}{\lambda}\right)^{2} F^{2} \cdot G^{2} \cdot H \cdot R \\
& G=\frac{\cos \left(\frac{a k}{2} \alpha_{f}\right)}{1-\left(\frac{\alpha_{f} k a}{\pi}\right)^{2}}, \quad F=\frac{2 \sin \left(\beta_{f} k b / 2\right)}{\beta_{f} k b}, H=\frac{1-\alpha_{f}^{2}}{\gamma_{f}} \quad, \quad \gamma_{f}=\sqrt{1-\alpha_{f}{ }^{2}-\beta_{f}^{2}}
\end{aligned}
$$

$\alpha$ and $\beta$ are the propagation constants of the wave in the $x-$ and $y$ - directions. The dipole impedance in this periodic array is expressed as a series of Floquet's harmonics. The impedance of a cylindrical dipole is nearly equal to that of a strip dipole with the strip width equal to 4 times the cylindrical dipole radius [10, ch.4].

The propagation constant of a natural surface wave is obtained as the zero of Eqn.(9), namely

$\mathrm{Zp}(\alpha, \beta)=0$

In order to find the real propagation constants $\beta$ of the surface waves in the y direction (propagation constant in $\mathrm{x}$ - direction, $\alpha=0$ ), we make a scan for the impedance $\mathrm{Z}_{\mathrm{p}}$ versus $\beta$. Fig. 5 shows the real and imaginary parts of dipole active impedance against the real propagation constant $\beta$, for array dimensions: $d_{x}=0.41 \lambda, d_{y}=.23 \lambda, a=0.39 \lambda, b=0.012 \lambda$. The behavior of the impedance in the figure is related to the grating lobe circles of the array [1], whose centers in the beta direction are at $n \lambda / d_{y}$, with unit radius. From Fig. 5 it is found that for values of $\beta$ within the grating lobe circles the real part of impedance is finite since radiation occurs in these regions. In the region between the grating lobe circles, the real part of impedance $=0$ since $\beta=\sin \theta>1$, which correspond to invisible space where no radiating space waves exist. This behavior repeats for the different grating lobes. In Fig. 5 the imaginary part has two zeroes (two roots) at two points; one 
at $\beta>1$ and another root at $\beta p \lambda / d_{y}-1$, and these roots repeat periodically between the different grating lobe circles. The propagation constants (roots) of the main two surface waves lie between the main-beam unit-radius grating lobe circle (in the grating lobe diagram) and the next grating lobe circle, whose center is located at $\lambda / \mathrm{d}_{\mathrm{y}}$. The discussion of Ref. $[2,8]$ do not point to roots other than the one near $\beta=1$. As the separation distance $d_{y}$ increases the region between the grating lobe circles decreases and the imaginary part does not attain zero values, so no real surface waves can propagate. A program for computing the complex surface wave propagation constants was made to search for the roots $\beta$ of Eqn. (10) ( $\alpha=0$ for propagation in the $y$ - direction) using Muller's method [11]. Eqn. (10) means that current wave with propagation constant $\beta$ can propagate on the array surface when the antennas terminals are short circuited.

In Eqn. (10) the propagation constant $\gamma_{\mathrm{f}}$, of the Floquet's harmonics in the $\mathrm{z}$ - direction, normal to the array plane, appears as a square root whose proper sign should be used. For the surface waves the propagation factor of the Floquet's harmonics is:

$$
e^{-j \alpha_{f} x} e^{-j \beta_{f} y} e^{-j \gamma_{f} z}
$$

Eqn. (10) shows that the propagation constants $\alpha_{\mathrm{f}}, \beta_{\mathrm{f}}$ of the Floquet's harmonics are $\alpha+\frac{\lambda}{d_{x}} m^{\prime}, \beta+\frac{\lambda}{d_{y}} n^{\prime}$, where $\mathrm{m}^{\prime}, \mathrm{n}^{\prime}$ run from minus infinity to infinity. The propagation constant $\gamma_{\mathrm{f}}$ is given by:

$\gamma_{f}=\sqrt{1-\alpha_{f}^{2}-\beta_{f}^{2}}$

According to the values of $\alpha_{\mathrm{f}}, \beta_{\mathrm{f}}$, the value of $\gamma_{\mathrm{f}}$ is chosen such that:

If $1>\alpha_{f}^{2}+\beta_{f}^{2} \Rightarrow \gamma_{f}$ is real (radiating harmonic)

If $\alpha_{f}^{2}+\beta_{f}^{2}>1 \Rightarrow \gamma_{f}$ is (-ve imaginary)

The latter condition corresponds to a wave decaying away from the array plane (surface wave).

Figure 6 shows the propagation constants $\beta$ of the surface waves propagating in the $y$ - direction, with the variation of the distance $d_{y}$ (normalized w.r.t. wavelength). The figure shows two real surface wave roots, the smaller one corresponds to the one obtained in Ref. [2, 8] and the greater one is another root not mentioned in these references. With small spacing the propagation constant of the main surface wave is nearly the free space one. As the distance $\mathrm{d}_{\mathrm{y}}$ increases, the two roots approach each other (since the grating lobe circles approach each other) until they become equal at $d_{y}$ $=0.39 \lambda$. This behavior is similar to that given in Ref. [4] for surface waves on the modulated reactance surface. If $d_{y}$ is increased beyond $0.39 \lambda$ in Fig. 6, no real roots exist. In this case leaky wave roots are searched with the increase of $d_{y}$, where the two surface wave roots merge into a leaky wave root.

For leaky waves, the propagation constant $\beta$ becomes complex, and the sign of the 
complex propagation constant $\gamma$ of the Floquet's harmonics in the direction normal to the array plane is taken to correspond to an outgoing wave. Such harmonics may decay or grow in the direction normal to the array plane $[4,5]$. The harmonics which grow are improper, however they can be incorporated in the solution since their fields exist in angular sectors where the field decays away from the surface [4].

The attenuation constant of the leaky wave (imaginary part) is found to be negligible, of the order of $10^{-6}$ or $10^{-7}$.

\section{Effects of different parameters on the surface wave and leaky wave propagation constants}

Figure 7 and Fig. 8 show the surface waves propagation constants as a functions of the dipole length ' $a$ ' and the separation distance ' $\mathrm{d}_{\mathrm{y}}$ ', where the curves are presented in the form of the propagation constants versus the distance ' $\mathrm{d}_{\mathrm{y}}$ ' with a certain value of $\mathrm{d}_{\mathrm{y}} / \mathrm{a}$ in each curve. It can be seen that as $a / d_{y}$ increases, the surface wave roots merge into a single root at a smaller value of $d_{y}$, such that at the merging point, ' $a$ ' is of the order of $0.4 \lambda$.

Figure 9 shows the effect of increasing the separation distance in the $\mathrm{x}$-direction to be equal to $10.4 \lambda$, which corresponds approximately to the linear Yagi array as studied in [3], where the elements in X-direction are now far from each other.

\section{Surface waves on arrays with source impedance or load impedance}

When the dipole is fed from a source with internal impedance or when a plane wave is incident on the array of dipoles with loads, the surface waves become attenuated due to the losses in the loads, i.e. the propagation constants become complex. Under loaded conditions, the source-free solution no longer corresponds to setting $Z_{p}=0$, Eqn.(10), to obtain the propagation constants, but rather the total impedance equals zero,

$$
Z_{p}(\beta)+Z_{\text {load }}=0
$$

This condition means that current wave with propagation constant $\beta$ can propagate on the array surface when the antennas terminals with loads are short circuited.

Figure 10 shows the current distribution, $25 \times 25$ elements array of cylindrical dipole, $\mathrm{d}_{\mathrm{x}}=0.41 \lambda, \mathrm{d}_{\mathrm{y}}=0.23 \lambda, \mathrm{a}=0.39 \lambda$, radius $=0.003 \lambda$, using $\mathrm{Z}_{\text {load }}=100$ ohms for an incident plane wave at $\theta_{0}=45^{\circ}$. The calculated normalized surface wave propagation constant from the interference pattern, Eqn.(8), gives $\beta=1.14$, and the root of the first surface wave gives $\beta=1.097-\mathrm{j} 0.156$, whose real part agrees reasonably with that calculated from the interference pattern. At the right of Fig. 10 an interference pattern is seen whose period is $2 \mathrm{~d}_{\mathrm{y}}$, which corresponds to a propagation constant $\beta=2.88$ when considered to interfere with the incident wave $\left(\beta=\mathrm{k} \sin \theta_{0}=0.707\right)$, or $\beta=3.27$ when considered to interfere with the first surface wave ( $\beta=1.097)$, and the second surface wave propagation constant is calculated from the characteristic equation to be $\beta=3.25$ - j0.156, whose real part agrees reasonably with that calculated from the interference pattern with the first surface wave. Thus, the interference pattern shows the excitation of the two surface waves. 


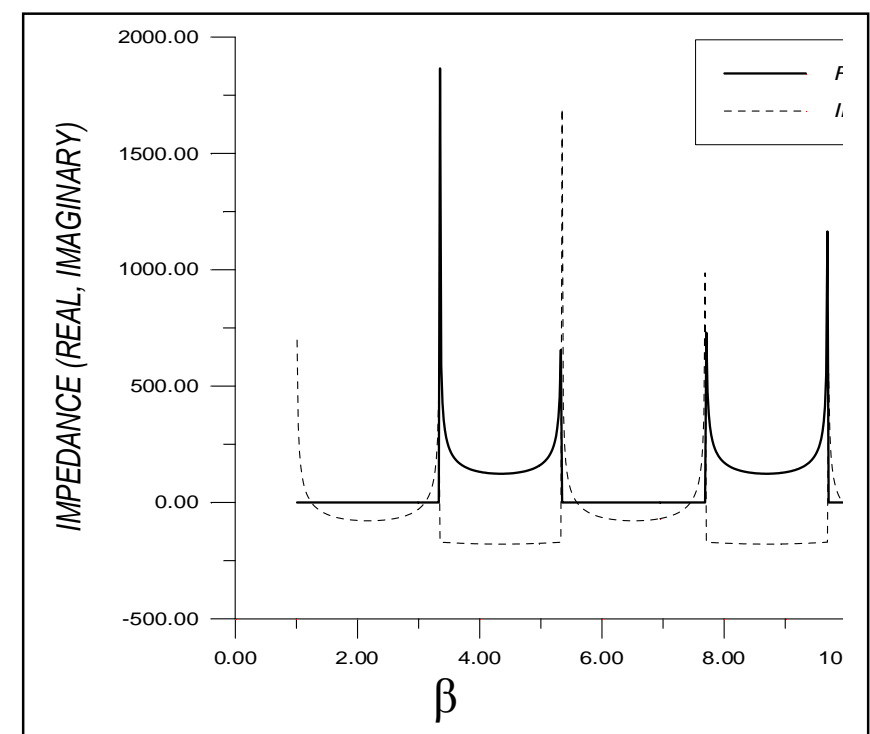

Fig. 5 Dipole active impedance in a planar infinite array, $d_{x}=0.41 \lambda$, $\mathrm{d}_{\mathrm{y}}=0.23 \lambda, \mathrm{a}=0.39 \lambda, \mathrm{b}=0.012 \lambda, \alpha=0$. (Impedance function has roots).

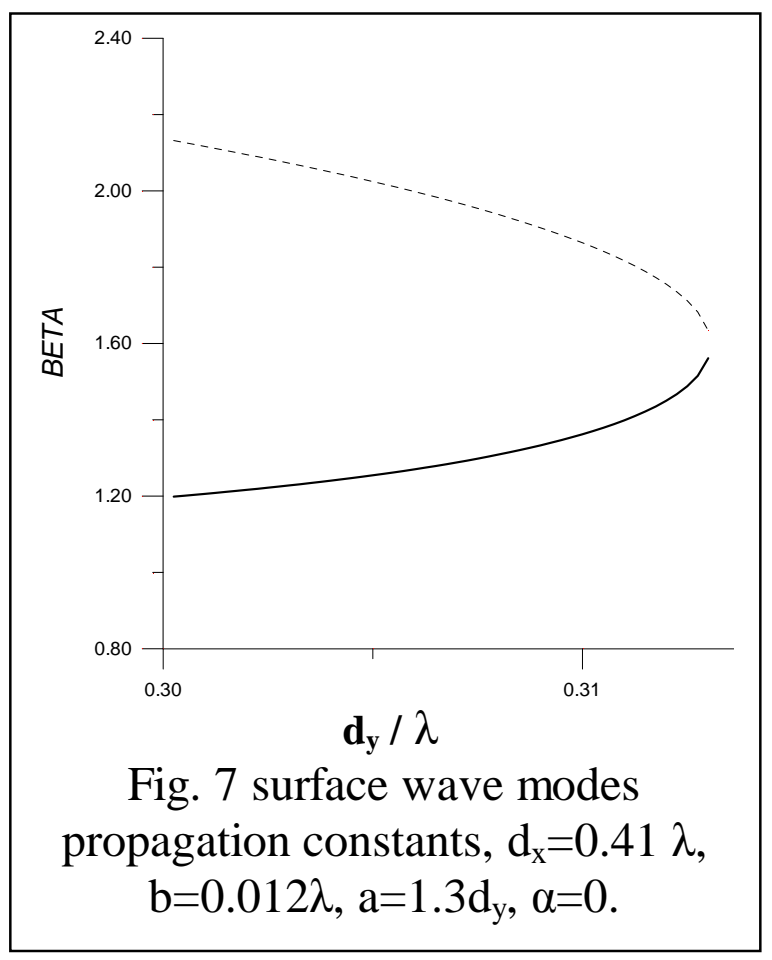

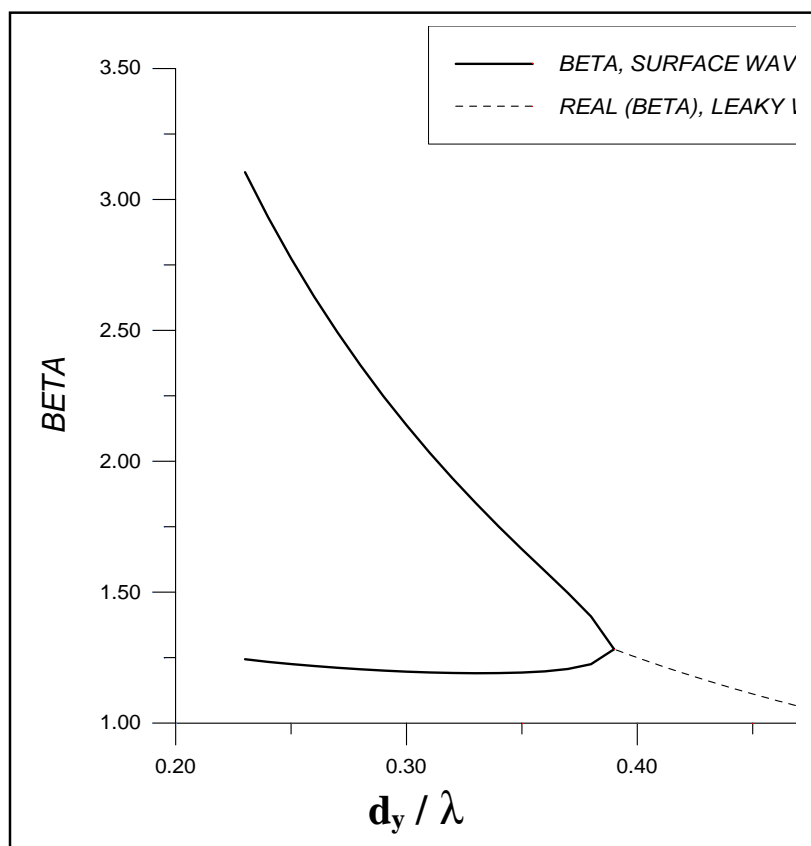

Fig. 6 Surface and leaky wave modes propagation constants, $d_{x}=0.41 \lambda$, $\mathrm{d}_{\mathrm{v}}=0.23 \lambda, \mathrm{a}=0.39 \lambda$,

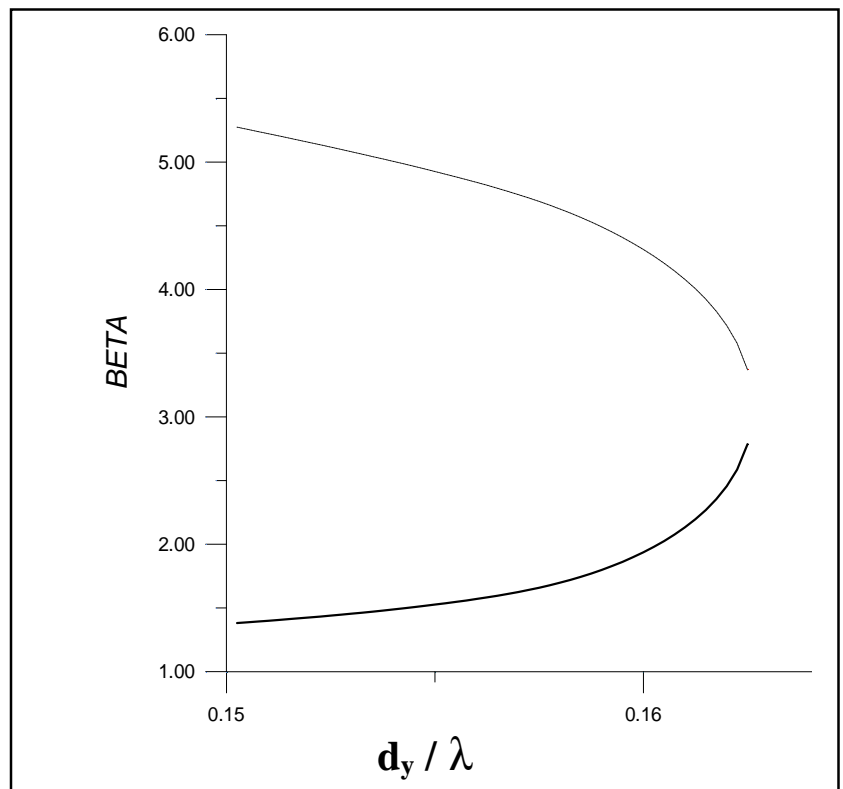

Fig. 8 surface wave modes propagation constants, $d_{x}=0.41 \lambda$, $h=0.017 \lambda . \quad a=7.6 d_{\ldots} . \alpha=0$. 


\section{Arravs with leaky waves}

Concerning Fig. 4 for the element impedances in case of scanning at $\theta_{0}=60^{\circ}$, the interference pattern is found to correspond to interference between the space wave with $\beta=\mathrm{k} \sin \theta_{0}$, and a traveling wave with propagation constant approximately equal to $\mathrm{k}$, with small attenuation constant. This traveling wave can not be a surface wave since the separation distance is $0.5 \lambda$, thus a leaky wave is expected rather than a surface wave. Fig. 11 shows the leaky wave propagation constant versus the load impedance for an array with resonant elements and $\mathrm{d}_{\mathrm{y}}=0.39 \lambda$. In fact, it was difficult to trace the root for larger values of $\mathrm{d}_{\mathrm{y}}$ because it required taking very small increments of $\mathrm{d}_{\mathrm{y}}$. Actually, the inverse of Eqn.(11) is more appropriate to take as the characteristic equation since the condition $\beta=1$, expected for the leaky wave from the interference patterns, Fig. 4, corresponds to an angle $\theta_{0}=90^{\circ}$, where $\beta=\sin \theta_{0}=1$. For an infinite array the dipole impedance at scan angle $\theta_{0}=90^{\circ}$ is infinite $[1,5]$. Thus we expect the value of $Z_{p}$ for this leaky wave to be infinity and the condition of the leaky waves in the presence of $Z_{\text {load }}$ to be:

$$
\mathrm{Z}_{\mathrm{p}}(\beta)+\mathrm{Z}_{\mathrm{load}}=\infty
$$

which defines also a source-free characteristic wave of the structure, i.e. a wave with propagation constant $\beta$ can propagate on the array surface when the antennas terminals are open circuited. In this case a finite load impedance has nearly no effect on the propagation constant.

Fig. 12 shows the propagation constant of the leaky mode satisfying Eqn.(12), whose real part equals unity, thus this mode can interpret the interference curves of Fig. 4.

Surface waves can not propagate on dipole arrays in the E-plane (collinear dipoles). The appropriate condition for leaky wave propagating in the E-plane is

$$
\mathrm{Z}_{\mathrm{p}(\alpha)}+\mathrm{Z}_{\mathrm{load}}=0
$$

This condition is used since at scan angle $\theta_{0}=90^{\circ}, \alpha=\sin \theta_{0}=1$ (as required for the leaky wave) and the element active impedance $Z_{p}$ of the infinite array is small [1, 5]. Fig. 13 shows the corresponding complex propagation constants of these leaky waves versus the load resistance. 

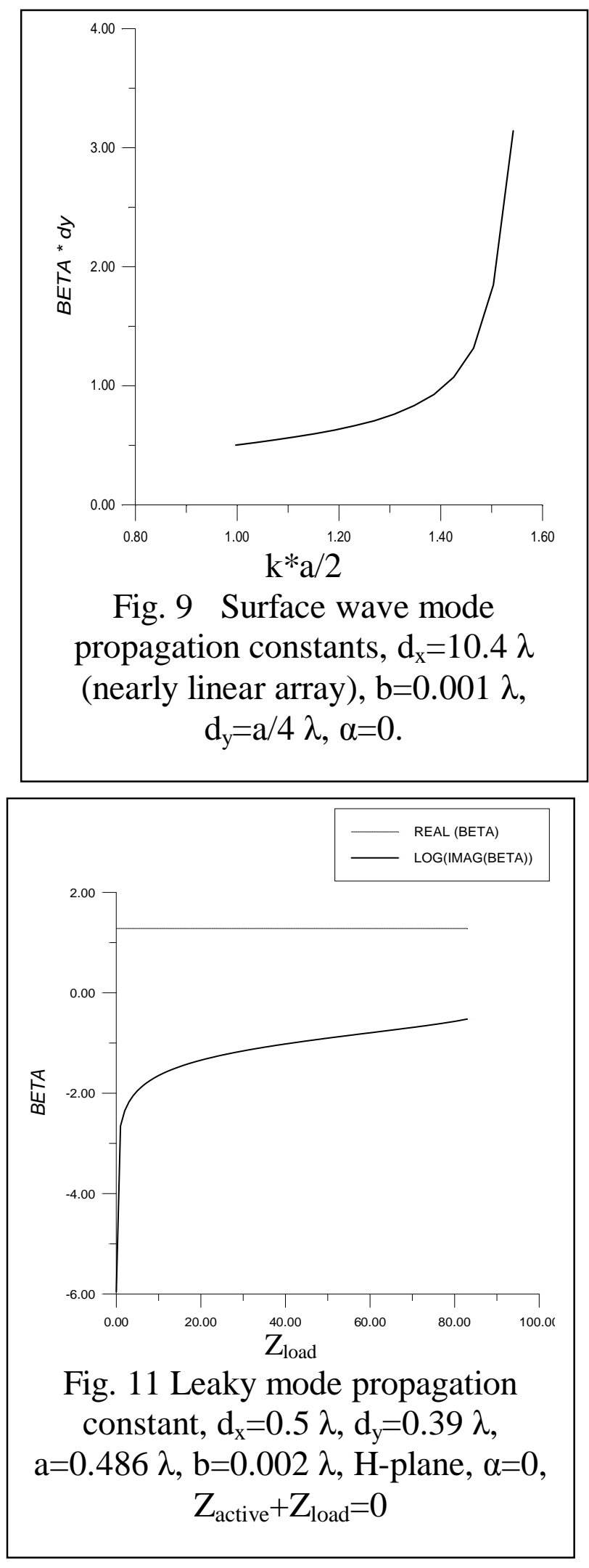

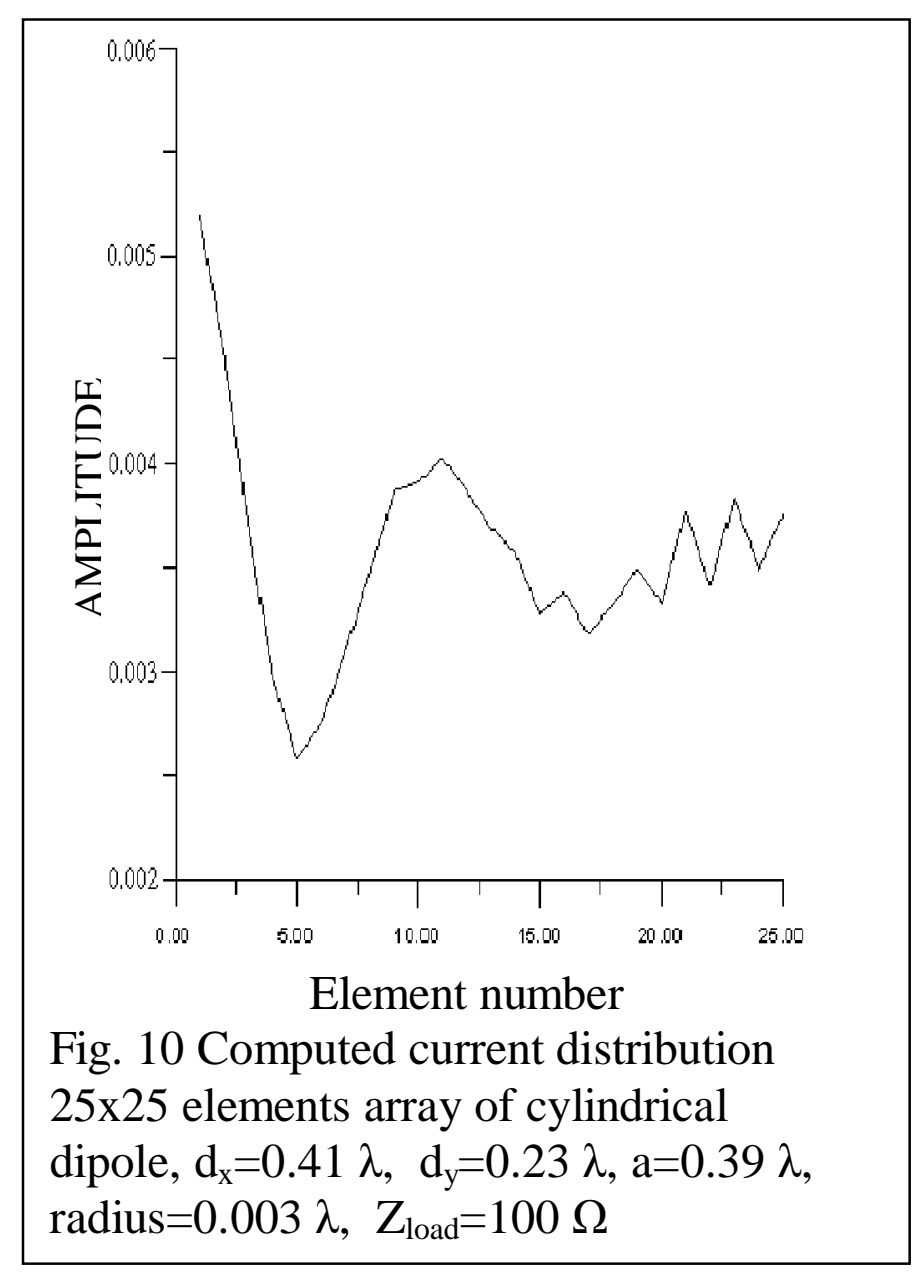



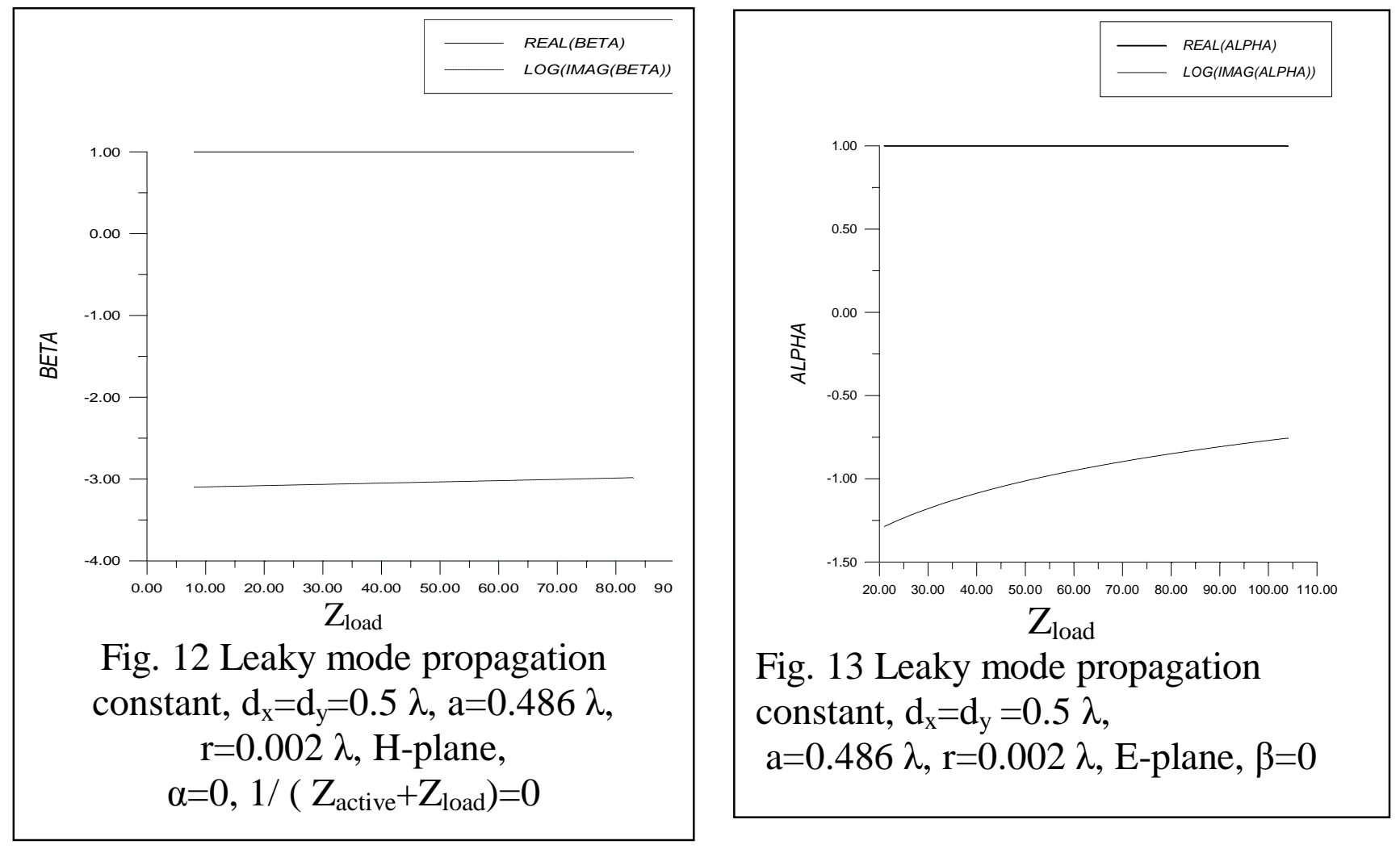

\section{Conclusions:}

Planar dipole arrays are periodic structures which can support traveling surface or leaky waves. The condition found to obtain the complex propagation constants of such natural source-free waves is that the corresponding element (plus the source or load) impedance in an infinite array is zero or infinite. The type of the traveling wave; whether a surface wave or a leaky wave, depends on the dipole length, the separating distances and the direction of propagation; whether along or perpendicular to the dipole directions. It is found that for the case of short non-resonant elements with small separation, more than one surface wave can propagate in the $\mathrm{H}$ - plane on the array surface, otherwise leaky waves propagate. Results were obtained for the propagation constants of the surface and leaky waves for different dipole lengths (short and resonant), element spacings (up to half wavelength), load or source impedance and direction of propagation (in E- or Hplane). The obtained propagation constants of the surface and leaky waves interpret reasonably the interference patterns found for the currents or impedances on the finite array surface between these traveling waves and the forced scanned wave excited by the generators (or the wave corresponding to an incident plane wave) 


\section{References:}

[1] R. C. Hansen," Phased Array Antennas", John Wiley, 1998

[2] B. A. Munk , "Finite Antenna Arrays and FSS", Wiley , 2003

[3] L. Shen, "Numerical analysis of wave propagation on a periodic linear array", IEEE Trans. Antennas \& Propagation, Vol. AP-19, Mar. 1971, pp.289-292.

[4] Hessel, "General Characteristics of Traveling- Wave Antennas", Chapter 19 "Antenna Theory II", R.E. Collin, F.J. Zucker (Ed.), 1969, McGraw Hill

[5] D.F. El-Hossary, E.A. El-Diwany, H.H. El-Tamaly, " Antenna Arrays and Their Applications in Electronic Warfare ", PH. D, thesis Minia University, 2007

[6] D.F. El-Hossary, E.A. El-Diwany, H.H. El-Tamaly, "Active impedances of elements in pencil beam and fan beam planar finite phased arrays of dipoles With low side lobe level " This issue .

[7] E.C. Jordan, K. G. Balmain, "Electromagnetic Waves and Radiating Systems", Prentice Hall, 1983.

[8] D. S. Janning and B. A. Munk, "Effects of surface waves on the currents of truncated periodic arrays", IEEE Trans. Antennas and Propagation, Vol.50, No. 9, Sep. 2002, pp.1254-1265.

[9] A. Ishimaru, R.Coe, G. Miller and P.Geren, "Finite periodic structure approach to large scanning array problems" IEEE Trans. Antennas and Propagation, Vol. AP-33, no.11, Nov.1985, pp. 1213 -1220.

[10] C. Johnson, H. Jasic (Ed.), "Antenna Engineering Hand Book", McGraw Hill, 1984 .

[11] S.D. Conte, C. de Boore, "Elementary Numerical Analysis", 1980, McGraw Hill 